\title{
Predicting Secondary-Dendrite Arm Spacing of the Al-4.5wt\%Cu Alloy During Unidirectional Solidification
}

\author{
Alexandre Furtado Ferreira ${ }^{*}$, José Adilson de Castro ${ }^{a}$, Leonardo de Olivé Ferreira ${ }^{b}$ \\ ${ }^{a}$ Graduate Program on Metallurgical Engineering and Mechanical Engineering, Federal Fluminense \\ University - UFF, Volta Redonda, RJ, Brazil \\ ${ }^{b}$ Universidade Federal do ABC-UFABC, Santo André, SP, Brazil
}

Received: March 9, 2015; Revised: August 8, 2016; Accepted: September 7, 2016

\begin{abstract}
Mechanical properties and performance of metallic materials depend on their microstructures. In order to develop engineering materials that match prescribed criteria and to enable design with multifunctional materials, it is essential to be able to predict their microstructural patterns, such as size, shape, and spacing of the dendritic structures observed in solidified metals. In the cases of metallic alloys, which present dendritic structure, the mechanical properties of foundry products depend mainly on the primary- and secondary-arm. Therefore, it is important, in a computational simulation of the solidification processes, to use reliable methods to correlate the thermal parameters with secondarydendrite arm spacing. This study presents a numerical model for prediction of secondary-arm spacing as a function of thermal parameters (cooling rates and local solidification time). Spacing of the arms for a binary alloy is numerically predicted using a phase-field model. Secondary dendrites calculated by phase-field model, they are similar to the ones found in experiments investigation of solidification in Al-Cu alloys. Arm spacing predicted in the present work, when compared with the experimental results, showed good agreement. Its estimation takes place at the late stage of growth. The effect of physical properties (partition coefficient $\left(k_{e}\right)$, diffusion in the liquid $\left(D_{L}\right)$ and diffusion in the solid phase $\left(D_{S}\right)$ ) on secondary-arm spacing is systematically investigated by phase-field model. With the help of numerical results for $\mathrm{Al}-4.5 \mathrm{wt} \% \mathrm{Cu}$ alloy, the applicability of the phase-field model to the estimation of secondary-dendrite arm spacing during unidirectional solidification is demonstrated.
\end{abstract}

Keywords: $\mathrm{Al}-\mathrm{Cu}$, dendrite, Phase-Field method, simulation, solidification

\section{Introduction}

Solidification is the main phenomenon taking place during casting. This, in turn, has long been known as a relatively inexpensive means for producing metal goods. Nowadays, a sizable portion of the concepts and methods developed over the years in support of the research into solidification phenomena can be implemented successfully and economically on the industrial scale. Noticeable improvement can thereby be achieved, insofar as the quality of the pieces manufactured by solidification is concerned. For this reason, solidification studies are not only mandatory; they are, indeed, a powerful industrial tool. When it comes to conventional technologies, thorough understanding and control of the solidification process opens wide perspectives in terms of its economic potential, since it provides the shortest distance from metal input to final product.

In-the-mold solidification of a metal, opposite to what might at first be surmised, is not a "passive" process in any way. On the contrary, the metal undergoes a liquid-to-solid transformation of a very dynamic nature. In its course, events occur - like the nucleation and growth of dendritic structures - which, in the absence of a tight control, may

\footnotetext{
* e-mail: furtado@metal.eeimvr.uff.br
}

compromise the final output or even halt the manufacturing process altogether. Such events can originate several types of material heterogeneities, which drastically affect the metallurgical quality of the final product.

On the other hand, aluminium alloy castings are widely used in many industries such as the automotive and aerospace ones. Owing to their low density as compared to ferrous alloys, aluminum alloys are very useful whenever lightweight and moderate strength are required of the castings.

The chemical, mechanical, and physical properties of cast and wrought metal products are in no small measure determined by the microstructure formed during solidification. Secondary-dendrite arm spacing has been observed to have a significant impact upon the yield strength, ultimate tensile strength, and elongation of cast products ${ }^{1}$. On the other hand, both the corrosion resistance of metallic alloys depends strongly on the solidification microstructural arrangement (secondary-arm spacing), as indicated by Osório et al. ${ }^{2}$ The correlation of corrosion behavior and mechanical properties with microstructure parameters can be very useful for planning solidification conditions. Knowledge of the effects of the secondary-dendrite arm spacing on the characteristics of a cast product has allowed the aluminium casting industry to effectively troubleshoot, develop, and improve different alloys. 
Understanding and modeling of dendritic growth has remained a central theme in solidification research for many years. Here, it should be noted that understanding the solidification process is of paramount importance because the resulting microstructure determines the properties of the materials.

In the past, knowledge about dendritic structures was based on experiments and idealized theoretical models. On the other hand, phase-field models are known to be very powerful in describing non-equilibrium dendritic evolution. They tend to be very efficient from the number-crunching standpoint on account of the fact all the governing equations are written for the whole domain, making no distinction between the interface and the solid and liquid phases. Furthermore, direct tracking of the interface position is not needed during simulation of the solidification process. Notice that the interface morphology of the solidification front, as calculated by our phase-field model, reproduces the known patterns of the dendritic structure. The state of the domain is represented by a distribution of the single variable known as "order parameter" or "phase-field variable." In the present paper, the solid state is represented by a phase-field variable equal to +1 , while, in the liquid region, its value is 0 (zero). The region in which it changes progressively from 0 to +1 is defined as the liquid/solid interface, the region where solidification effectively happens.

Phase-field models have, over recent years, garnered wide acceptance, given their ability to simulate the solidification process in the presence of a complicated solid/liquid interface. These models can be found in a collection of papers in the open literature. They were developed mainly for studying solidification of pure materials, being subsequently extended to the solidification of binary, ternary and quaternary alloys. The first models focused on pure materials. Such was the case, for example, of Kobayashi ${ }^{3}$, Kim et al. ${ }^{4}$, and Ferreira et al. ${ }^{5}$ Binary alloys were then treated, e.g., in Oguchi and Suzuki ${ }^{6}$ and Ode et al. ${ }^{7}$ Next, ternary alloys were attacked by Ferreira and de-Olivé Ferreira ${ }^{8}$ and by Ode et al. ${ }^{9}$ A little more recently, quaternary alloys were dealt with in Salvino et al. ${ }^{10}$ Usually, phase-field works employ divided differences to obtain solutions to the equations. Moreover, in the case of alloys, dendrite growth is considered at constant temperature or constant cooling rate.

In this paper, a phase-field model is resorted to in order to analyze the growth of secondary dendrite arms in $\mathrm{Al}-4.5 \mathrm{wt} \% \mathrm{Cu}$ binary alloys. We adopt the phase-field model proposed by Ode et al. ${ }^{7}$ and use a divided-differences method for solution of the equations, keeping a constant cooling rate during numerical simulation of the solidification. Alterations in secondary-arm spacing are induced both by changing local solidification time and cooling rates. The secondary-arm spacing is evaluated with different values of partition coefficient and solute diffusivities in the liquid and solid regions.

\section{Governing Equations}

In phase-field models, the state of the domain is customarily represented by a distribution of the single variable known as the "order parameter" or "phase-field variable," here represented by the Greek letter $\phi$. As previously indicated, in this study, it is assumed that the solid state corresponds to a value of +1 for the order parameter, while, in the liquid region, $\phi$ is taken to be 0 . The region through which $\phi$ decreases from +1 to 0 is defined as the solid/liquid interface. For simulation of microstructures in binary alloys during solidification, we used two equations: one for solute concentrations, the other for the phase field itself. Following Ode et al. ${ }^{7}$, the first equation takes the form

$$
\frac{1}{M} \frac{\partial \phi}{\partial t}=\varepsilon^{2} \nabla^{2} \phi-W g^{\prime}(\phi)+\frac{R T}{V_{m}} h^{\prime}(\phi) \ln \left[\frac{\left(1-c_{S}^{e}\right)\left(1-c_{L}\right)}{\left(1-c_{L}^{e}\right)\left(1-c_{S}\right)}\right],
$$

where " $\ell n$ " stands for the principal argument of the natural logarithm of the fraction function within the square brackets. As for the phase-field equation, those authors propose

$$
\frac{\partial c}{\partial t}=\nabla\left\{D(\phi)\left[\begin{array}{l}
(1-h(\phi))\left(1-c_{L}\right) c_{L}+ \\
h(\phi)\left(1-c_{S}\right) c_{S}
\end{array}\right] \nabla \ln \left(\frac{c_{L}}{1-c_{L}}\right)\right\},
$$

where $h(\phi)=\phi^{2 \cdot}(3-2 \phi), g(\phi)=\phi^{\cdot 2 \cdot}(1-\phi)^{2}$, and the subscripts $S$ and $L$ stand for solid and liquid, respectively. $M$ and $\varepsilon$ are phase-field parameters; $D(\phi)$ is the solute diffusion coefficient. These phase-field parameters are related to the interface energy, $\sigma$, whereas the interface width, $2 \lambda$, is the distance over which $\phi$ changes from 0.1 to 0.9 . Notice, furthermore, that $M$ is also related to the kinetic coefficient, $\beta$, defined to be the inverse of the usual linear kinetic coefficient, $\mu_{. k .}$. From Salvino et al. ${ }^{10}$, these are obtained as

$$
\begin{gathered}
\sigma=\frac{\varepsilon_{0} \sqrt{W}}{3 \sqrt{2}}, \quad(3) \\
2 \lambda=2.2 \sqrt{2} \frac{\varepsilon_{0}}{\sqrt{W}}, \quad(4) \\
\beta=\left(\frac{V_{m}}{R T}\right)\left(\frac{\sigma}{\varepsilon^{2} M}-\frac{\varepsilon_{0}}{D_{i} \sqrt{2 W}} \xi\left(c_{S}^{e}, c_{L}^{e}\right)\right) \frac{m_{e}}{1-k_{e}}, \quad \\
\xi\left(c_{S}^{e}, c_{L}^{e}\right)=\frac{R T}{V_{m}}\left(c_{L}^{e}-c_{S}^{e}\right)^{2} \\
\times \int_{0}^{1} \frac{(1-h(\phi)) h(\phi)}{(1-h(\phi))\left(1-c_{L}^{e}\right) c_{L}^{e}+h(\phi)\left(1-c_{S}^{e}\right)} \frac{d \phi}{(1-\phi) \phi}
\end{gathered}
$$

where $m_{e}$ is the slope of the liquidus line at equilibrium, $k_{e}$ is the equilibrium partition coefficient, and $D_{i}$ is the diffusion coefficient in the interface region. For the binaryalloy system, we use the same parameters shown in the literature, Ode et al. ${ }^{7}$ In addition, $T$ is the temperature, $W$ represents interface energy, and $V_{m}$ is the molar volume. - Equations (1) and (2) were solved numerically. They were discretized on uniform grids using an explicit finite scheme. 
Anisotropy is introduced in the phase-field model as follows:

$$
\varepsilon(\theta)=\varepsilon_{0}\left\{1+\delta_{\varepsilon} \cos \left(j \cdot\left(\theta-\theta_{0}\right)\right)\right\},
$$

where $\delta_{\varepsilon}$ gauges the anisotropy. The value $j$ controls the number of preferential growth directions. For example, with $j=0$, we shall be looking at a perfectly isotropic case, while $j=4$ is indicative of a dendrite with four preferential growth directions. Orientation of the maximum-anisotropy interface is identified by the $\theta_{0}$ constant of Eq. (7), $\theta$ being the angle between the direction of the phase-field gradient and the reference axis of the system.

\section{Numerical Calculations}

For the simulation, Eqs. (1) and (2) are discretized using an explicit finite difference scheme. To obtain the twodimensional dendrite shape, a large number of calculation meshes are required. Therefore, two-dimensional phasefield simulations are restricted not only by computational efficiency but also by usable-memory size. To simulate growth of an asymmetrical dendrite, it is necessary to introduce a noise term on the right-hand side of Eq. (1), the phase-field equation. A usual expression for this noise, as indicated by Ferreira et al. ${ }^{13}$, is

$$
\text { noise }=16 \operatorname{ar} \phi^{2}(1-\phi)^{2} \text {, }
$$

with $r$ a random number between -1 and +1 . The " $a$ " parameter is the noise amplitude. Maximum noise corresponds to $\phi=0.5$, at the center of the interface, whereas at $\phi=0$ (liquid region) and $\phi=1$ (solid region) there occurs no noise. That is to say, noise is generated at the interface. The parameters used in the phase-field model, obtained from physical properties of the material, were derived from Eqs. (3) to (6). The boundary condition adopted for the phase-field model $(\phi)$ in this work is a zero-flux condition. The small mesh size, $\Delta x=\Delta y$, is necessary so as to accurately calculate the phase field, which varies markedly, only nears the interface, remaining constant in both the liquid and solid regions.

\section{Results and Discussion}

Table 1, below, presents the physical properties of the binary alloy $(\mathrm{Al}-4.5 \mathrm{wt} \% \mathrm{Cu})$ utilized in the simulations that follow. As just mentioned, the parameters employed in the phase-field model, obtained from physical properties of the material, were derived from Eqs. (3) to (6). Table 2 presents these parameters. Like was also mentioned right above, the boundary condition adopted for the phase-field model $(\phi)$ in this work is a zero-flux condition.

The governing equations involve seven unknown values. Three of them are phase-field parameters. $W$ and $\varepsilon_{0}$ are determined by solving Eqs. (3) and (4) simultaneously. Since phase-field mobility is a function of the temperature, it
Table 1: Physical properties of Al-4.5wt $\% \mathrm{Cu}$ alloys, Oguchi and Suzuki ${ }^{6}$.

\begin{tabular}{lcc}
\hline Property & $\mathrm{Cu}$ & $\mathrm{Al}$ \\
\hline Initial concentration, $C_{0}[\% \mathrm{~mol}]$ & $1.96 \times 10^{-2}$ & \\
Partition coefficient, $k_{e}$ & 0.14 & \\
Slope of liquidus line, $m_{e}\left[\mathrm{~K} \cdot \mathrm{mol}^{-1}\right]$ & 640 & \\
Diffusivity in the solid region, $D_{S}\left[\mathrm{~m}^{2} \cdot \mathrm{s}^{-1}\right]$ & $3 \times 10^{-13}$ & \\
Diffusivity in the liquid region, $D_{L}\left[\mathrm{~m}^{2} \cdot \mathrm{s}^{-1}\right]$ & $3 \times 10^{-9}$ & \\
Molar volume, $V_{m}\left[\mathrm{~m}^{3} \cdot \mathrm{mol}^{-1}\right]$ & & $1.095 \times 10^{-5}$ \\
Melting temperature, $T_{m}[\mathrm{~K}]$ & & 933 \\
Interface energy, $\sigma\left[\mathrm{J} \cdot \mathrm{m}^{-2}\right]$ & & 0.093 \\
\hline
\end{tabular}

Table 2: Computational parameters.

\begin{tabular}{ll}
\hline Parameter & Value \\
\hline Magnitude of anisotropy, $\delta_{\varepsilon}$ & 0.03 \\
Coefficient of phase-field gradient energy, $\varepsilon_{0}$ & $1.055 \times 10^{-3}$ \\
{$\left[\mathrm{~J} \cdot \mathrm{m}^{-1}\right]^{1 / 2}$} & \\
Height of the double-well potential, $W\left[\mathrm{~J} \cdot \mathrm{m}^{-3}\right]$ & $673.2 \times 10^{3}$ \\
Time step, $\Delta t[\mathrm{~s}]$ & $1.5 \times 10^{-6}$ \\
Mesh size, $\Delta x=\Delta y[\mathrm{~m}]$ & $1.5 \times 10^{-7}$ \\
Noise amplitude, $a$ & 0.03 \\
\hline
\end{tabular}

should be calculated along with the temperature, during the computation. The values of solute concentration in liquid, $c_{L}$, and solid, $c_{S}$, are also required; They are determined from Eq. (2) according to the values of $\phi$ and $c$ at each point and every time step. The governing equations, (1) and (2) above, are solved numerically, using a finite-difference scheme. In the calculations, the system temperature is uniform and continuously decreased with a constant cooling rate from the initial temperature, $T_{0}$, which is slightly lower than the liquidus temperature of $\mathrm{Al}-4.5 \mathrm{wt} \% \mathrm{Cu}$ alloy.

\subsection{Growth of Secondary-Dendrite Arms}

The phase-field model is used to calculate the dendrites, by imposing a constant temperature gradient in an undercooled melt system. Simulation of dendrite evolution is carried out disregarding the energy equation and instead imposing the following linear temperature profile:

$$
T=T_{0}-(T t)
$$

where $T_{0}$ is the initial temperature and $T t$ represents a constant value for the cooling rate.

The growth and selection of secondary-dendrite arms with constant cooling rate equal to $19.84 \mathrm{~K} / \mathrm{sec}$ for $\mathrm{Al}-4.5 \mathrm{wt} \% \mathrm{Cu}$ are shown in Figure 1. All the initial solid seeds are growing, but the shapes of the arms are different from each other due to the noise imposed via Eq. (8).

The advanced arms in Figure 1(a) grow preferably and other arms stop advancing or slightly melt back due to the 

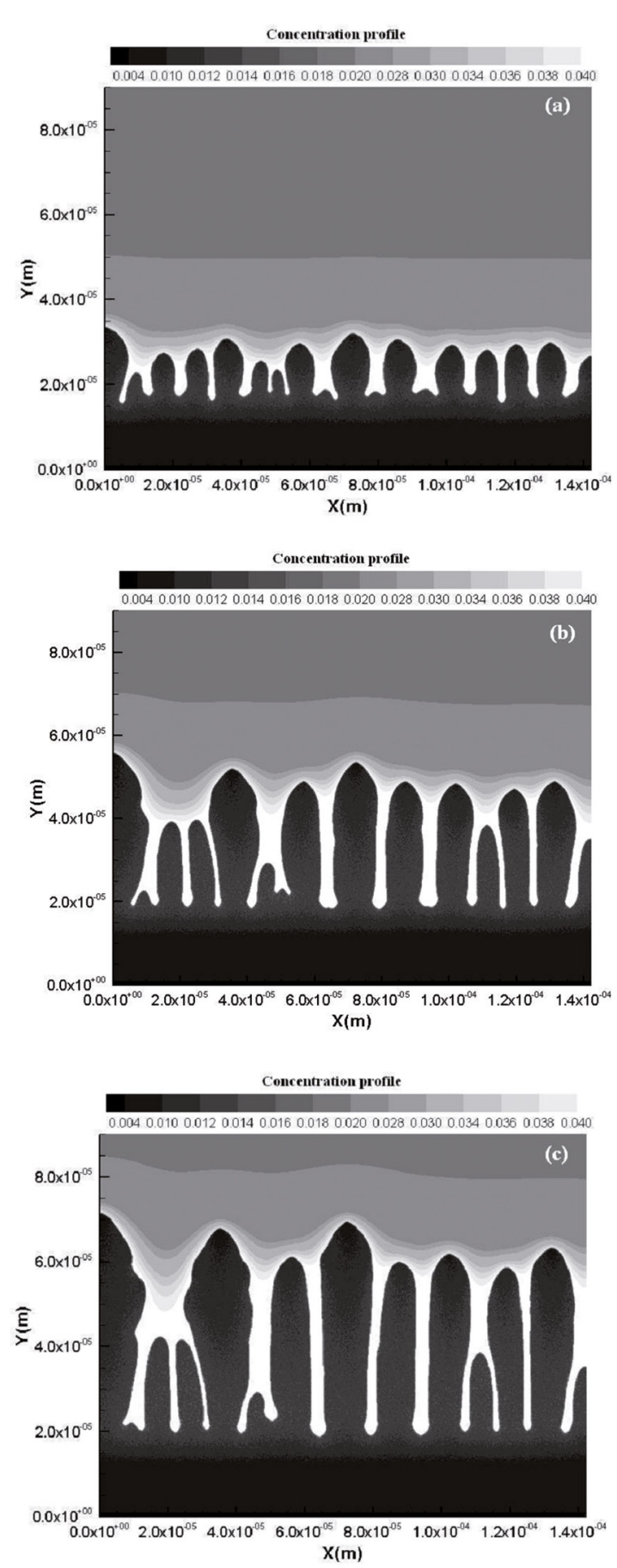

Figure 1: Growth of secondary arms for Al-4.5\%wtCu: (a) Early stage of arm growth, $t=5.139 \times 10^{-2} \mathrm{sec}$; (b) arm growth and selection, $t=8.37 \times 10^{-2} \mathrm{sec}$; (c) coarsening of arm, $t=0.105 \mathrm{sec}$.

curvature effect, Figure 1(b). After selection of the secondary arms, a coarsening of the selected arms is observed in Figure 1(c). Thus, our phase-field calculation displays arm selection and the determination of the arm spacing that occurs sequentially. In the phase-field model, initial solid seeds are physically selected to grow or shrink. Since the phase-field calculation physically reproduces competitive growth of the arms from the perturbed interface, the final arm spacing is independent of initial condition.

System temperature was reduced constantly from the initial temperature, $T_{0}$. Mesh size and time step used were, respectively, $1.5 \times 10^{-7} \mathrm{~m}$ and $1.5 \times 10^{-6} \mathrm{sec}$.

\subsection{Prediction of the Arm Spacing}

Secondary-dendrite arm spacing also depends on the local solidification time, according to theoretical and experimental works referenced by Garcia ${ }^{12}$. Said time is the time required for a particular region to solidify, once nucleation has begun; In other words, it is the time for solidification completion in this region. It depends on the characteristics of the metal being cast, like its density, heat capacity, and fusion heat. For the unidirectional solidification case, Garcia $^{12}$ expresses the local solidification time is as

$$
t_{S}=\Delta T / \dot{T}
$$

where $\Delta T$ is the interval for solidification and $\dot{T}$ represents the cooling rate. On the other hand, for the secondary-arm spacing, the same author proposes the following approximate function of the local solidification time:

$$
\lambda_{2}=12.1 t_{S}^{0.33}
$$

This equation is experimentally obtained for $\mathrm{Al}-4.5 \mathrm{wt} \% \mathrm{Cu}$ alloy. The relationship between secondary-dendrite arm spacing and local solidification time for our Al-Cu alloy is depicted in Figure 2. Arm spacing is estimated at the late stage of growth.

Results from model and experimental data are plotted for comparison. The secondary dendrite arm spacing is in good agreements with the experimental data, Garcia ${ }^{12}$. The results show that both the local solidification time and the secondary-arm spacing increased over the process.

The relationship between secondary-dendrite arm spacing and cooling rate for the Al-Cu alloy we analyzed appears in Figure 3. The results show the effect of cooling rate on the microstructural features of that alloy.

The solidification process was studied using cooling rates ranging from 5 to $45 \mathrm{~K} / \mathrm{sec}$. One can see that increasing the cooling rate influences the reduction of the secondary-arm spacing. The secondary arms develop well and arm spacing becomes narrow with an increase of cooling rates. This happens because, under such an increase, interface stability is reduced. This, in turn, is due to an accompanying decrease of the phase-field mobility in Eq. (5).

The relationship between secondary-arm spacing, $\lambda_{2}$, and cooling rate, $\dot{T}$, is obtained by means of a correlation analysis of the phase-field results displayed in Figure 3, ultimately leading to

$$
\lambda_{2}=37.94 \dot{T}^{0.25},
$$




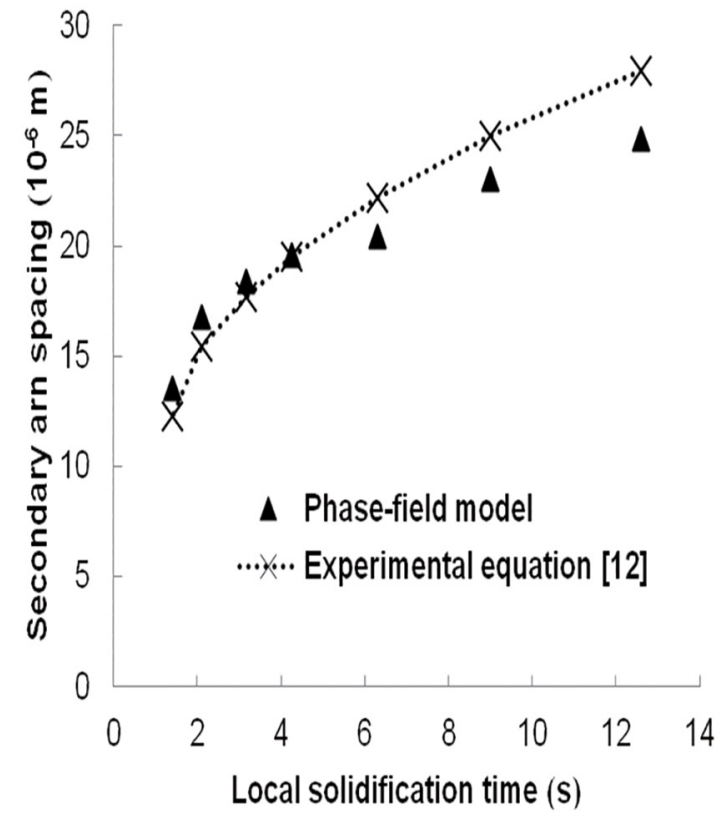

Figure 2: Secondary-arm spacing versus local solidification time for our $\mathrm{Al}-4.5 \mathrm{wt} \% \mathrm{Cu}$ alloy.

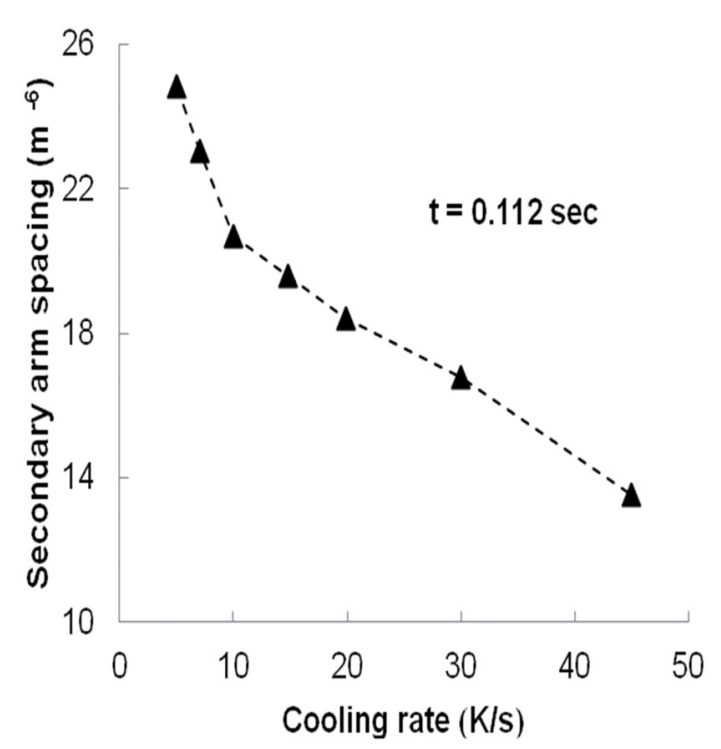

Figure 3: Secondary-arm spacing versus cooling rate for Al$4.5 \mathrm{wt} \% \mathrm{Cu}$ alloy, with a solidification time of $0.112 \mathrm{sec}$.

The effects of the physical properties $\left(k_{e}, D_{L}\right.$ and $\left.D_{S}\right)$ on secondary-dendrite arm spacing are shown in Figures 4-6. The spacing is evaluated with different values of partition coefficient $\left(k_{e}\right)$ and of solute diffusivities in liquid $\left(D_{L}\right)$ and solid $\left(D_{S}\right)$ in the Al-Cu binary alloy.

During the solidification process, there occurs a distribution of solutes at the phase interface. Distribution of solutes between the solid and liquid phases is characterized by the equilibrium partition coefficient. Knowledge of this coefficient is important for the study of segregation of both micro- and macro-inhomogeneities in alloys. It constitutes

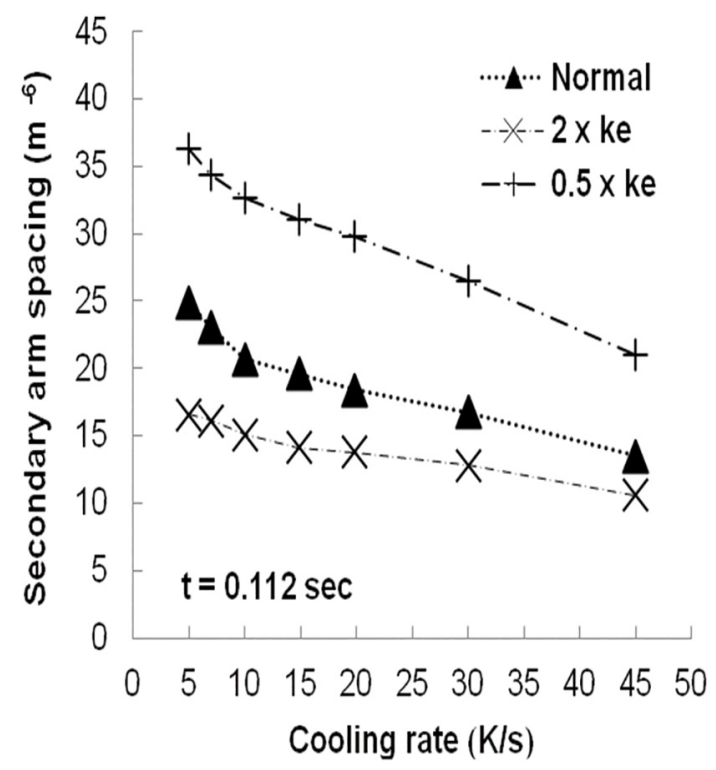

Figure 4: Effect of the partition coefficient $\left(k_{e}\right)$ on the secondarydendrite arm spacing, with a solidification time of $0.112 \mathrm{sec}$.

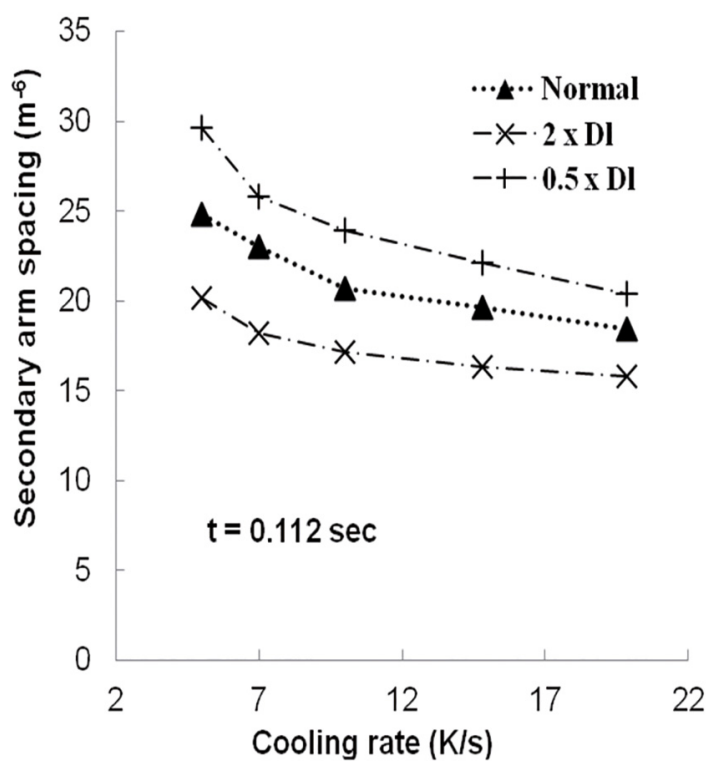

Figure 5: Effect of solute diffusion in the liquid $\left(D_{L}\right)$ on secondarydendrite arm spacing with a solidification time of $0.112 \mathrm{sec}$.

the main material parameter for the production of highly pure materials by such refining processes as zone melting and directional crystallization.

A thorough understanding of the way dendrites occurs during solidification should be of great importance for properties of the material and in designing post-casting processes such as heat-treatments. The constant equilibrium partition coefficient is determined from the $\mathrm{Al}-\mathrm{Cu}$ phase diagram. On the other hand, the casting process takes place under non-equilibrium conditions. In such a case, there 


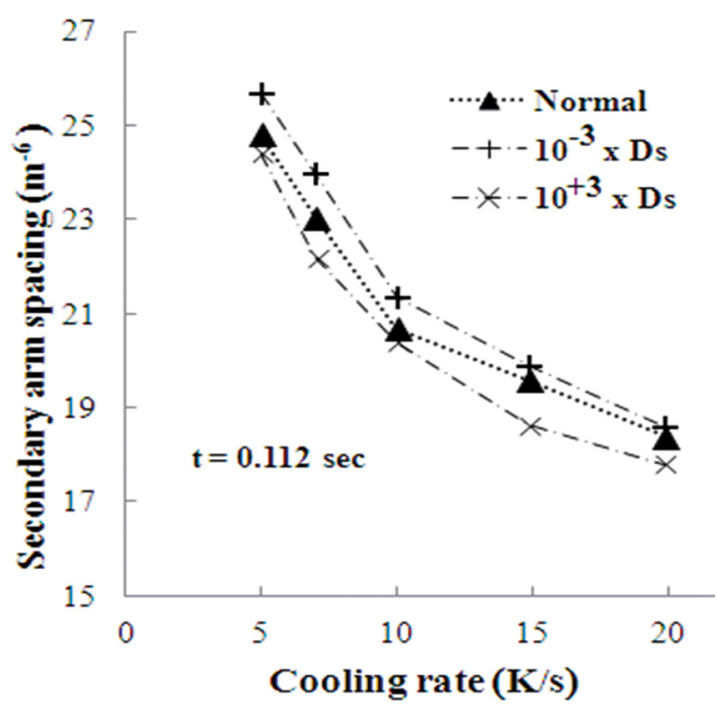

Figure 6: Effect of solute diffusion in the solid phase $\left(D_{S}\right)$ on secondary-dendrite arm spacing with a solidification time of $0.112 \mathrm{sec}$.

are significant deviations of the partition coefficient from its equilibrium value. In this work, we tested the effect of the partition coefficient on secondary-arm spacing, as shown in Figure 4.

Secondary-arm spacing is evaluated with different values of partition coefficient. One can see in Figure 4 that, when the partition coefficient is large, the slope of the line becomes shallow. On the other hand, changes in cooling rate do not alter significantly the secondary-arm spacing. However, when the partition coefficient is small, the slope of the line becomes larger. The effect of that coefficient on secondary-dendrite arm spacing rests thus demonstrated. The secondary-arm spacing is seen to decrease faster for cooling rates between 5 and $10 \mathrm{~K} / \mathrm{sec}$; it then gradually diminishes for cooling rates up to $45 \mathrm{~K} / \mathrm{sec}$. In this situation, the partition coefficient is termed "normal." Therefore, for both normal and small partition coefficients, the changes in arm spacing are more significant than for partition coefficient equal to $2 \times k_{e}$.

Diffusivities in the liquid state are much higher than diffusivities in the solid one. In the case of metals, diffusivities in the two phases differ by a factor of 100 to $10^{+9}$, depending on the type of material. The high atomic mobility of most metals just above their melting temperatures, with diffusivities on the order of $10^{-9} \mathrm{~m}^{2} \mathrm{sec}^{-1}$, is one of the most characteristic properties of liquid metals. The study of diffusion processes in melts is vital for an understanding of liquid dynamics, nucleation, and dendritic growth. Diffusion coefficients are an essential input to the modeling of microstructure evolution. The study of diffusion in liquid metals is of great interest because of various scientific and technological reasons. The knowledge of diffusion coefficients plays an important role in the design of metallurgical and solidification processes such as happens in the casting industry.

The transport properties of liquid metals, together with thermodynamic information, can provide an experimental basis for theories of the liquid state. In this work, we analyze the effect capability of the solute diffusion in liquid $\left(D_{L}\right)$ and solid $\left(D_{S}\right)$ on arm spacing; The results are shown in Figures 5 and 6, respectively. In Figure 5, one can see the solute diffusivity in the liquid region change the arm spacing. The most significant changes occur for a solute diffusivity equal to $0.5 \times D_{L}$ as opposed to the $2 \times D_{L}$ curve. The effect, on arm spacing, of solute diffusivity in the solid is depicted in Figure 6. The secondary-arm spacing is evaluated with different values of solute diffusivities in the solid region. For a situation dubbed "normal," $10^{-3} \times D_{S}$, and then, $10^{+3} \times D_{S}$. Figure 6 show that solute diffusivities in the solid do not affect the secondary-arm spacing. In all cases studied, the solidification time is $0.112 \mathrm{sec}$ (Figures 4-6).

The solidification parameters of interest for modeling dendritic growth (such as partition coefficient and solute diffusivities) can be obtained by using thermodynamic calculations. In this study, we investigate the effect of physical properties on the phase-field model, i.e., the phase-field mobility is evaluated with different values of partition coefficient and solute diffusivities in liquid and solid. Figures $7 \& 8$ display the physical properties that affect the phase-field mobility.

Figure 7 exhibits the effect of different values of the partition coefficient on phase-field mobility. When that coefficient is large, the phase-field mobility also becomes large; in other words, the partition coefficient significantly changes phase-field mobility. Figure 8 , in turn, shows the effect, on phase-field mobility, of solute diffusivity in the liquid. Once again, the same conclusion is drawn (as discussed in the previous case); i.e., solute diffusivity in the liquid region contributes to induce changes in phase-field mobility. One can see a seeming tendency toward a linear behavior in the plot of phase-field mobility. Such linearity could, however, be a mere consequence of the short time interval considered here.

Solute diffusivity in the solid region does not appear to affect either the phase-field mobility, Figure 9, or the secondary-dendrite arm spacing, Figure 6. On the other hand, when physical properties do affect phase-field mobility, Figs. $7 \& 8$, the secondary-dendrite arm spacing is changed, too, Figs. $4 \& 5$. This seems to suggest a strong connection between secondary-arm spacing and phase-field mobility. The dependence of phase-field mobility on the physical properties can be explained by Eq. 6. In that equation, one can see the relation between them. 


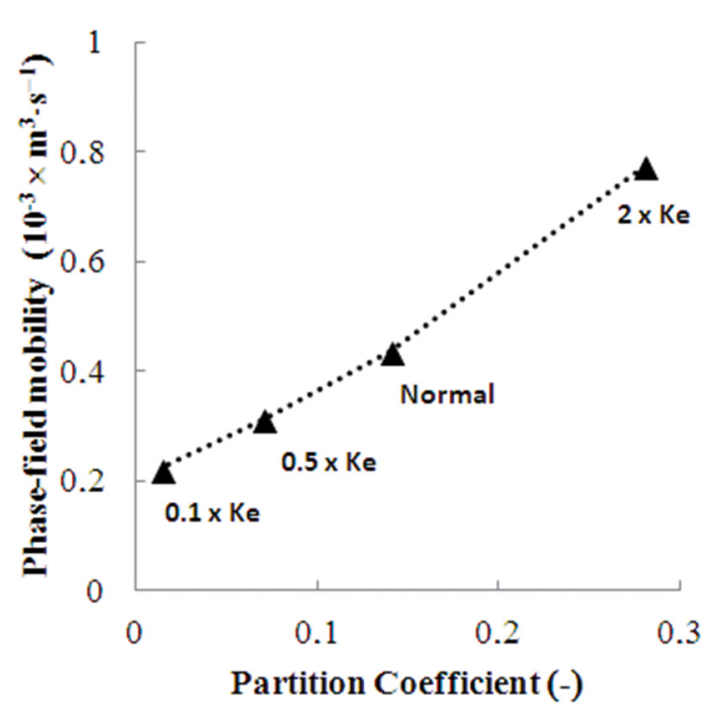

Figure 7: Phase-field mobility $(M)$ versus partition coefficient $\left(k_{e}\right)$.

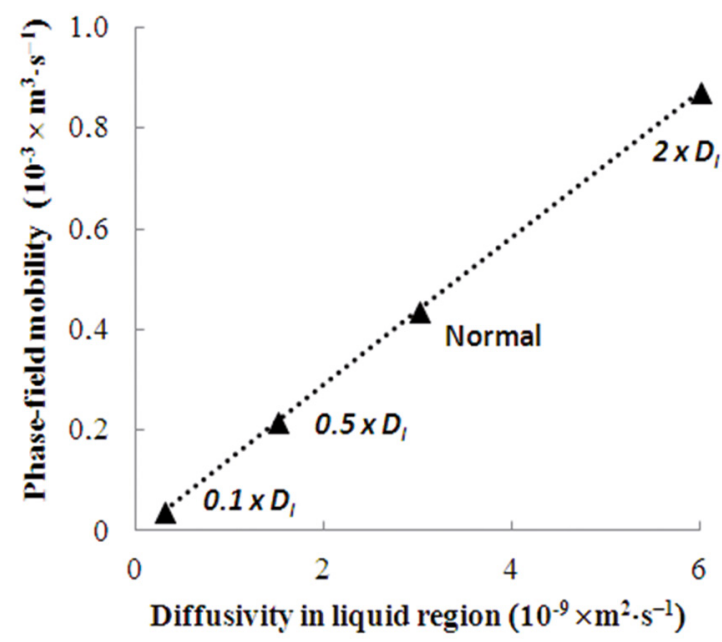

Figure 8: Phase-field mobility $(M)$ versus diffusivity in the liquid $\left(D_{L}\right)$.

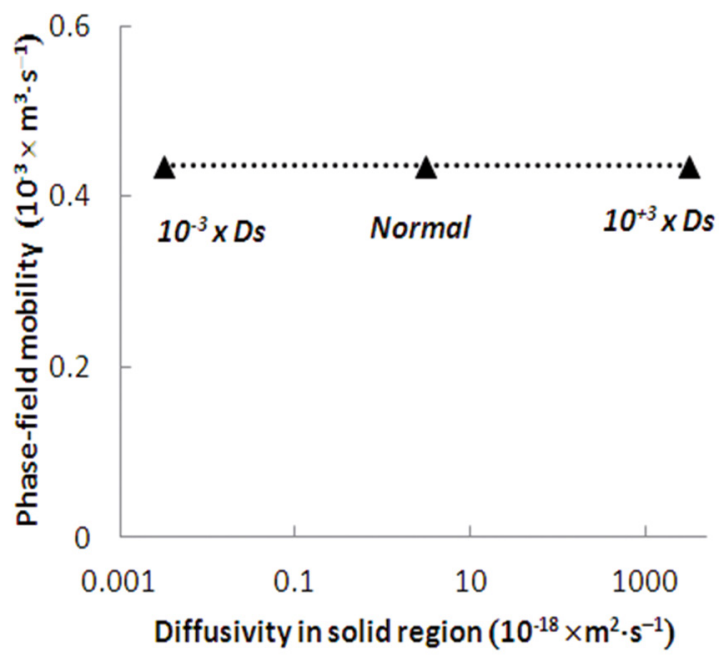

Figure 9: Phase-field mobility $(M)$ versus diffusivity in the solid $\left(D_{S}\right)$.

\section{Conclusions}

We analyze dendrite growth in a two-dimensional domain. The relations between materials properties and model parameters are presented. Two-dimensional simulations produced dendrite arms that are similar to the ones found in experiments reported in the literature. A phase-field model for a binary alloy is applied to the numerical prediction of secondary-dendrite arm spacing in an Al-4.5wt \%Cu alloy. Said spacing is estimated at the late stage of growth. Arm selection and competitive growth between arms are successfully reproduced. The calculated secondary-arm spacing is in good agreement with a previously reported experimental equation with local solidification time. The effect of the cooling rate on secondary-arm spacing is examined. Our results show that, an increasing the cooling rate, the secondary-arm spacing decreases. This takes place because the secondary-arm spacing depends on the competitive growth of arms during solidification. As the cooling rate increases, system temperature decreases faster, and the competitive growth between secondary arms is less intense. The effect of physical properties on secondarydendrite arm spacing is systematically investigated. Partition coefficient and diffusivity in liquid affect both secondary-arm spacing and phase-field mobility. On the other hand, diffusivity in the solid region induces no alteration of either the spacing or the phase-field mobility. Our numerical results show that the secondary-arm spacing varies significantly when physical properties affect the phase-field mobility.

\section{References}

1. Rao DH, Tagore GRN, Janardhana GR. Evolution of Artificial Neural Network (ANN) model for predicting secondary dendrite arm spacing in aluminium alloy casting. Journal of the Brazilian Society of Mechanical Sciences and Engineering. 2010;32(3):276-281.

2. Osório WR, Goulart PR, Garcia A, Santos GA, Moura Neto C. Effect of dendritic arm spacing on mechanical properties and corrosion resistance of Al $9 \mathrm{Wt}$ Pct Si and Zn $27 \mathrm{Wt}$ Pct Al alloys. Metallurgical and Materials Transactions A. 2006;37(8):2525-2538.

3. Kobayashi R. Modeling and numerical simulations of dendritic crystal growth. Physica D: Nonlinear Phenomena. 1993;63(34):410-416.

4. Kim SG, Kim WT, Lee JS, Ode M, Suzuki T. Large scale simulation of dendritic growth in pure undercooled melt by phase-field model. ISIJ International. 1999;39(4):335-340.

5. Ferreira AF, Silva AJ, Castro JA. Simulation of the solidification of pure nickel via the phase-field method. Materials Research. 2006;9(4):349-356.

6. Oguchi K, Suzuki T. Phase-field simulation of free dendrite growth of aluminum- 4.5 mass\% copper alloy. Materials Transactions. 2007;48(9):2280-2284.

7. Ode M, Suzuki T. Numerical simulation of initial evolution of $\mathrm{Fe}-\mathrm{C}$ alloys using a phase-field model. ISIJ International. 2002;42(4):368-374. 
8. Ferreira AF, Ferreira LO. Microsegregation in Fe-C-P ternary alloys using a phase-field model. Journal of the Brazilian Society of Mechanical Sciences and Engineering. 2009;31(3):173-180.

9. Ode M, Kim SG, Suzuki T. Recent advances in the phase-field model for solidification. ISIJ International. 2001;41(10):1076-1782.

10. Salvino IM, Ferreira LO, Ferreira AF. Simulation of microsegregation in multicomponent alloys during solidification. Steel Research International. 2012;83(8):723-732.
11. Salvino IM, Jácome PAD, Ferreira AF, Ferreira IL. An analysis of the physical properties of multicomponent alloys on the simulation of solidification by phase-field model. Materials Science Forum. 2012;730-732:703-708.

12. Garcia A. Solidificação: Fundamentos e Aplicações. $2^{\mathrm{a}}$ ed. Campinas: Unicamp; 2007. 400 p.

13. Ferreira AF, Melo EG, Ferreira LO. Prediction of secondarydendrite arm spacing for binary alloys by means of a phasefield model. Steel Research International. 2014;86(1):58-64. 